\title{
HIV infection increases HCV-induced hepatocyte apoptosis
}

\author{
Jae Young Jang ${ }^{1,2}$, Run-Xuan Shao ${ }^{1}$, Wenyu Lin $^{1}$, Ethan Weinberg ${ }^{1}$, Woo Jin Chung ${ }^{1}$, Wei Lun Tsai ${ }^{1}$, \\ Hong Zhao ${ }^{1}$, Kaku Goto ${ }^{1}$, Leiliang Zhang ${ }^{1}$, Jorge Mendez-Navarro ${ }^{1}$, Nikolaus Jilg ${ }^{1}$, Lee F. Peng ${ }^{1}$, \\ Mark A. Brockman ${ }^{3}$, Raymond T. Chung ${ }^{1, *}$
}

${ }^{1}$ Gastrointestinal Unit, Department of Medicine, Massachusetts General Hospital, Harvard Medical School, Boston, MA 02114, USA; ${ }^{2}$ Institute for Digestive Research, Digestive Disease Center, Department of Internal Medicine, College of Medicine, Soonchunhyang University, Seoul, South Korea; ${ }^{3}$ Partners AIDS Research Center, Massachusetts General Hospital and Division of AIDS, Harvard Medical School, USA

Background \& Aims: HCV related liver disease is one of the most important complications in persons with HIV, with accelerated fibrosis progression in coinfected persons compared to those with HCV alone. We hypothesized that HCV-HIV coinfection increases HCV related hepatocyte apoptosis and that HCV and HIV influence TRAIL signaling in hepatocytes.

Methods: We analyzed the effect of HIV in JFH1-infected Huh7.5.1 cells. Apoptosis was measured by Caspase-Glo 3/7 assay and Western blotting for cleaved PARP. TRAIL, TRAIL receptor 1 (DR4), and 2 (DR5) mRNA and protein levels were assessed by real-time PCR and Western blot, respectively. We also investigated activation of caspase pathways using caspase inhibitors and assessed expression of Bid and cytochrome $C$.

Results: We found increased caspase 3/7 activity and cleaved PARP in JFH1 HCV-infected Huh7.5.1 cells in the presence of heat-inactivated HIV, compared to Huh7.5.1 cells infected with JFH1 or exposed to heat-inactivated HIV alone. Both DR4 and DR5 mRNA and protein expression were increased in JFH1infected cells in the presence of inactivated HIV compared to Huh7.5.1 cells infected with JFH1 or exposed to heat-inactivated HIV alone. Pancaspase, caspase- 8 , and caspase- 9 inhibition blocked apoptosis induced by HCV, inactivated HIV, and HCV plus inactivated HIV. A caspase-9 inhibitor blocked apoptosis induced by HCV, HIV, and HCV-HIV comparably to pancaspase and caspase- 8 inhibitors.

HCV induced the activation of Bid cleavage and cytochrome $C$ release. The addition of HIV substantially augmented this induction.

Conclusions: Our findings indicate that hepatocyte apoptosis is increased in the presence of HCV and HIV compared to HCV or HIV alone, and that this increase is mediated by DR4 and DR5 up-regulation. These results provide an additional mechanism for the accelerated liver disease progression observed in HCVHIV co-infection.

Keywords: HCV-HIV coinfection; Apoptosis; TRAIL receptor.

Received 31 March 2010; received in revised form 12 July 2010; accepted 16 July 2010; available online 29 September 2010

* Corresponding author. Address: Gastrointestinal Unit, Warren 1007, Massachusetts General Hospital, Boston, MA 02114, USA. Tel.: +1 617724 7562; fax: +1 6176430446

E-mail address: rtchung@partners.org (R.T. Chung).

Abbreviations: JFH-1, Japanese Fulminant Hepatitis 1; TRAIL, TNF-related apoptosis-inducing ligand; PARP, poly (ADP-ribose)polymerase; TGF- $\beta 1$, transforming growth factor beta1; DR4, Death receptor 4; DR5, death receptor 5.
C 2010 European Association for the Study of the Liver. Published by Elsevier B.V. All rights reserved.

\section{Introduction}

Hepatitis C virus (HCV) infects about 170 million people and is a leading cause of chronic liver disease worldwide [1,2]. It is a major cause of cirrhosis, a significant cause of hepatocellular carcinoma (HCC), and the leading reason for liver transplantation worldwide.

HCV and human immunodeficiency virus (HIV) frequently coexist because of their common routes of transmission, and liver disease caused by HCV has rapidly become one of the most important complications in persons with HIV [3].

Indeed, end-stage liver disease is the most frequent cause of death among HIV-infected hospitalized patients [4]. Although the cause of liver injury in HIV-infected individuals is likely multifactorial (i.e., due to coinfection and medications), a number of reports have documented histological liver abnormalities that occurred solely as a result of HIV infection [5,6]. Moreover, HIV-1 RNA [5] and p24 antigen have been isolated from livers of infected individuals [7], although direct evidence that HIV infects hepatocytes is lacking. These observations suggest that HIV may indirectly result in hepatocellular injury. The hallmark of HIV infection is depletion and exhaustion of the immune system.

Hepatocyte cell death by apoptosis is emerging as a fundamental component of virtually all acute and chronic liver diseases. The ensuing responses of cell repair, inflammation, regeneration, and fibrosis may all be triggered by apoptosis [811]. An increasing body of evidence from both experimental and clinical studies suggests that hepatocyte apoptosis may contribute to liver fibrogenesis. Engulfment of apoptotic bodies by hepatic stellate cells stimulates the fibrogenic activity of these cells and may be one mechanism by which hepatocyte apoptosis promotes fibrosis [12]. Furthermore, recent studies in chronic hepatitis $\mathrm{C}$ virus ( $\mathrm{HCV}$ ) infection demonstrate that hepatocyte apoptosis correlates with disease severity [13] and stage of fibrosis in patients with steatosis $[14,15]$.

Death receptors 4 and 5 (DR4 and DR5), also called tumor necrosis factor-related apoptosis-inducing ligand (TRAIL)-R1 and R2, are members of the tumor necrosis factor-receptor 
(TNFR) family $[16,17]$ and receptors for TNF-related apoptosisinducing ligand (TRAIL) $[18,19]$. TRAIL selectively induces apoptosis in cancer cells in vitro and in vivo with little or no toxicity toward normal cells [20-22]. DR4 and DR5 mediate TRAILinduced apoptosis through the formation of a death inducing signaling complex (DISC) containing the death receptor, adapter proteins such as Fas-associated death domain (FADD), and initiator caspases such as pro-caspase-8 [23] or pro-caspase-10 $[24,25]$. Consequently, pro-caspase- 8 or pro-caspase- 10 is activated by autoproteolytic processing, which then cleaves and activates downstream effector caspases, such as caspase-3 (extrinsic pathway). Additionally, the Bcl-2-interacting protein Bid is also cleaved by caspase-8 [26]. Truncated-Bid causes the loss of mitochondrial membrane potential and caspase- 9 cleavage, resulting in apoptosis. Thus, truncated-Bid is a mediator that transduces the pro-apoptotic signal from the extrinsic pathway to the intrinsic (mitochondrial) pathway (Fig. 1).

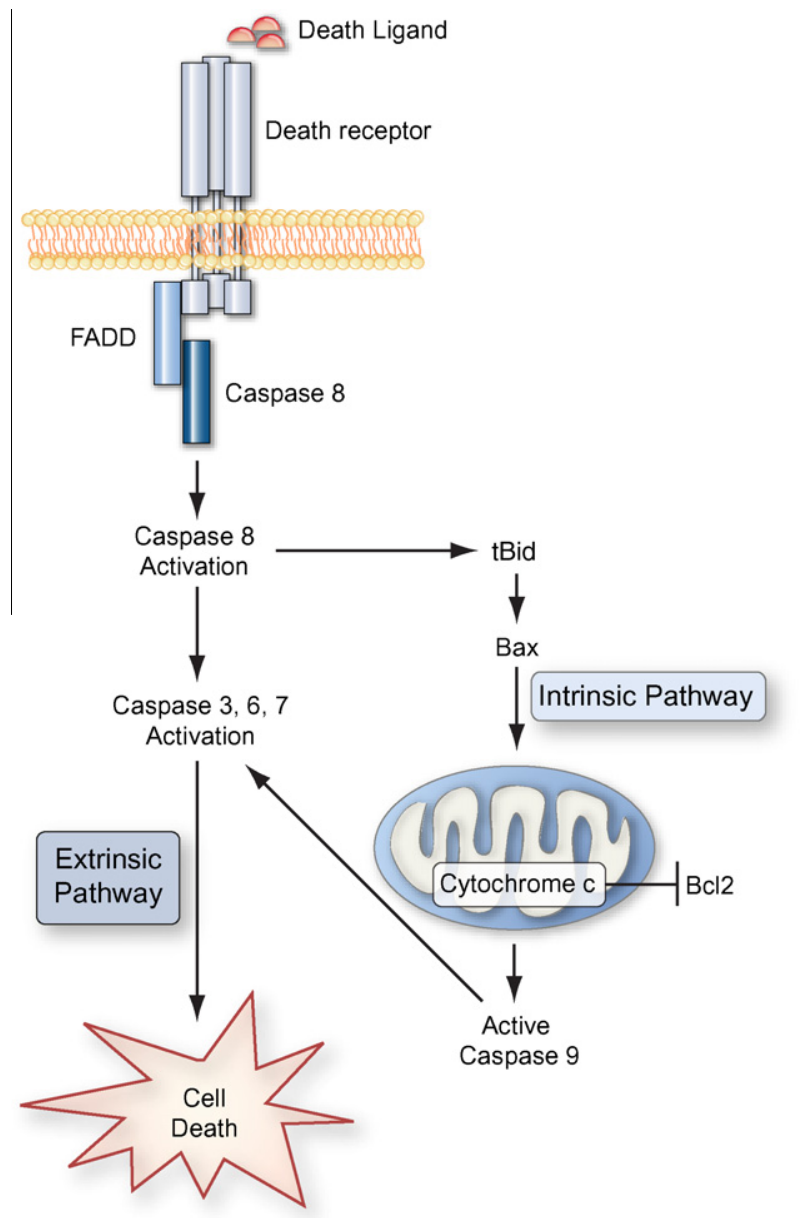

Fig. 1. Pathways of Cellular Apoptosis. Death receptor mediates death ligandinduced apoptosis through the formation of a death inducing signaling complex (DISC) containing the death receptor, adapter proteins such as the Fas-associated death domain (FADD), and initiator caspases such as pro-caspase- 8 or procaspase-10. Consequently, pro-caspase- 8 or pro-caspase-10 is activated by autoproteolytic processing, which then cleaves and activates downstream effector caspases, such as caspase-3 (extrinsic pathway). Additionally, the Bcl2 -interacting protein Bid is also cleaved by caspase- 8 . Truncated-Bid causes the loss of mitochondrial membrane potential and caspase- 9 cleavage, resulting in apoptosis. Thus, truncated-Bid is a mediator that transduces the pro-apoptotic signal from the extrinsic pathway to the intrinsic (mitochondrial) pathway.

\section{JOURNAL OF HEPATOLOGY}

We hypothesized that HIV coinfection augments hepatocyte apoptosis attributable to HCV and that this represents an additional mechanism of accelerated liver disease in coinfection. We tested this hypothesis using an infectious HCV cell culture model.

\section{Materials and methods}

Cell cultures and transfection

Huh7.5.1 cells (human hepatocellular carcinoma cells) were grown in Dulbecco's modified Eagle's medium supplemented with $10 \%$ fetal bovine serum (FBS). Japanese Fulminant Hepatitis 1 (JFH1) is a known tissue culture infectious strain of HCV. We transfected JFH1 RNA into Huh7.5.1 cells and collected the culture medium at $72 \mathrm{~h}$ post-transfection, as described previously [27]. We then inoculated naïve Huh7.5.1 cells with these culture supernatants, and confirmed JFH1 virus infection of newly inoculated cells by real-time PCR and Western blot for HCV RNA and core protein, respectively. NL4-3 is a CXCR4-tropic (T lymphocyte-tropic) HIV strain. BaL is a CCR5-tropic (macrophage-tropic) HIV strain. Heat-inactivated HIV (CXCR4 and CCR5-tropic virus) $\left(56^{\circ} \mathrm{C}\right.$ for $30 \mathrm{~min}$ ) was incubated with Huh7.5.1 cells or JFH1-infected Huh7.5.1 cells for $72 \mathrm{~h}$ at $1: 4$ dilution (11.25 ng/ $\mathrm{ml} \mathrm{p} 24$ for NL4-3; $8.75 \mathrm{ng} / \mathrm{ml} \mathrm{p} 24$ for BaL). Naïve Huh7.5.1 cells were seeded at a density of $2 \times 10^{5}$ cells/well in 6 -well plates $24 \mathrm{~h}$ before transfection. Infection of JFH1 and incubation with heat-inactivated HIV were performed simultaneously. Subsequently, cells were washed with PBS and collected $72 \mathrm{~h}$ later after transfection.

HIV stocks

Heat-inactivated HIV was prepared as previously described [28]. Laboratoryadapted HIV-1 strains were obtained from the NIH AIDS Research and Reference Reagent Program. A plasmid encoding the CXCR4-tropic virus NL4-3 was used to transfect HEK293T cells, and the supernatant virus was propagated using a CEMderived T-cell line that expresses CD4, CXCR4, and CCR5 (CEM-GXR) [28]. The CCR5-tropic virus BaL [29] was also propagated using CEM-GXR cells. Viral stocks were assayed for HIV-1 p24 using the Alliance p24 Antigen ELISA Kit (PerkinElmer, Waltham, MA). Previously, we found that the increase in HCV levels mediated by heat-inactivated CCR5-tropic HIV and CXCR4-tropic HIV was abrogated by pre-incubation with neutralizing antibody to CXCR4 and CCR5 [30]. These data provided evidence for the indirect effect of HIV virus on HCV infection signaling through engagement of its co-receptors on the surface of Huh7.5.1 cells rather than through direct infection of hepatocytes.

Real-time polymerase chain reaction quantification

Total cellular RNA was harvested using the RNeasy Kit (Qiagen, Valencia, CA) according to the manufacturer's protocol. Total cDNA was synthesized by reverse transcription using the GeneAmp RNA PCR Kit (Applied Biosystems, Branchburg, New Jersey). DR4, DR5, TRAIL and $\beta$-actin mRNA levels were quantified by realtime PCR. Human DR4 (GenBank\#: NM_003844), Human DR5 (GenBank\# NM_003842) and Human TRAIL (GenBank\# NM_003810) were measured using the following primers: DR4 sense primer CTGAGCAACGCAGACTCGCTGTCCAC; DR4 antisense primer-TCAAAGGACACGGCAGAGCCTGTGCCA; DR5 sense primerGGGAGCCGCTCATGAGGAAGTTGG; DR5 antisense primer-GGCAAGTCTCTCTCCCAGCGTCTC; TRAIL sense primer-AATCATCAAGGAGTGGGCATTC; TRAIL antisense primer-ATGACCAGTTCACCATTCCTCAA. Human $\beta$-actin sense primer-GCACTCTT CCAGCCTTCCT; human $\beta$-actin antisense primer AGGTCTTTGCGGATGTCCAC. $\beta$-Actin was used as a control for basal RNA levels. DR4, DR5, TRAIL, and $\beta$-actin levels were quantified by real-time PCR using the Bio-Rad IQ5 (Bio-Rad Laboratories, Hercules, CA). Finnzymes SYBR green I dye (New England Biolabs, Ipswich, MA) was used for detection, as previously described [31].

Protein sample preparation

At the time of harvest, cells were washed twice with phosphate-buffered saline, and whole-cell protein samples were extracted with lysis buffer $(50 \mathrm{mM}$ Tris- $\mathrm{HCl}$ $1 \mathrm{mM}$ EDTA, $50 \mathrm{mM} \mathrm{NaF}, 1 \mathrm{mM} \mathrm{Na}{ }_{3} \mathrm{VO}_{4}, 1 \%$ Triton X-100, $150 \mathrm{mM} \mathrm{NaCl}, 10 \mu \mathrm{g} / \mathrm{ml}$ aprotinin, phosphatase inhibitor cocktail, and protease inhibitor cocktail) (Roche Applied Science, Indianapolis, IN). 
Research Article

Western blotting

Proteins were separated by SDS-polyacrylamide gel electrophoresis (PAGE) with NuPAGE Novex Bis-Tris precast 4-12\% gradient gels (Invitrogen Life Science Carlsbad, CA) and transferred to nitrocellulose membranes. The primary antibodies used for Western blots were as follows: rabbit anti-cleaved PARP (1:1000)(Cel signaling Technology, Inc., Beverly, MA), mouse anti-DR4 (1:1000) (Abcam Inc. Cambridge, MA), rabbit anti-DR5, rabbit anti-TRAIL, mouse anti-Bid, rabbit anticytochrome $C$ (all at 1:1000, Cell signaling Technology, Inc., Beverly, MA), mouse anti-HCV core (1:1000)(Affinity BioReagents Inc., Golden, CO), and mouse antiactin $(1: 10,000)$ (Sigma Life Science and Biochemicals, St. Louis, MO). The secondary antibody was horseradish peroxidase-conjugated ECL donkey anti-rabbit IgC $(1: 2000)$ or sheep anti-mouse IgG $(1: 10,000)$ (Amersham Biosciences, Piscataway, $\mathrm{NJ})$. Chemiluminescent signals were detected using the ECL Western blotting detection Kit (Amersham Biosciences, Piscataway, NJ). Densitometry was performed using the Universal Hood II (Bio-Rad, Hercules, CA).

Poly (ADP-ribose) polymerase assay

PARP, a 116 kDa nuclear poly (ADP-ribose) polymerase, appears to be involved in DNA repair in response to environmental stress. This protein can be cleaved by many ICE-like caspases in vitro and is one of the main cleavage targets of caspase-3 in vivo. In human PARP, the cleavage occurs between Asp214 and Gly215, which separates the PARP amino-terminal DNA binding domain (24 kDa) from the carboxy-terminal catalytic domain (89 kDa). PARP helps cells to maintain their viability; cleavage of PARP facilitates cellular disassembly, and serves as a marker of cells undergoing apoptosis [32].

Caspase 3/7 activity assay

The Caspase-Glo ${ }^{\circledR}$ 3/7 Assay provides a homogeneous luminescent assay that measures caspase-3/7 activity. Naïve Huh7.5.1 cells were seeded at a density of $10^{4}$ cells/well ( $100 \mu \mathrm{l}$ of DMEM with $10 \% \mathrm{FBS}$ ) in 96 -well plates, $24 \mathrm{~h}$ before transfection. Infection of JFH1 and incubation with heat-inactivated HIV were performed simultaneously. Caspase-Glo 3/7 reagent (Promega Corporation, Madison. WI) was then added to each well, $72 \mathrm{~h}$ after transfection. The contents of the plate were gently mixed using a plate shaker at $300-500 \mathrm{rpm}$ for $30 \mathrm{~s}$. The cells were then lysed at room temperature for $1 \mathrm{~h}$. Luminescence activities (relative caspase 3 and 7 activities) were measured with a BioTek Synergy 2 Microplate Reader (Winooski, VT). We measured caspase-3/7 activities as a function of JFH1 concentration using the Caspase-Glo ${ }^{\circledR} 3 / 7$ Assay. We found that this assay sensitively detected apoptosis at very low concentrations of JFH1 (data not shown). Because of its sensitivity, we used caspase-3/7 activity as an apoptotic marker for this study.

Caspase inhibitors

Cells were preincubated with caspase inhibitors (pan-caspase inhibitor: Z-VADFMK $(20 \mu \mathrm{M})$, caspase-8 inhibitor: Z-IETD-FMK $(20 \mu \mathrm{M})$, caspase-9 inhibitor: Z LEHDFMK $(20 \mu \mathrm{M})$; all from R\&D Systems) for $6 \mathrm{~h}$ prior to the caspase-Glo $3 / 7$ assay. Caspase inhibitors were subsequently diluted in DMSO before use.

\section{SiRNA and transfection}

To further explore the relationship between apoptosis and TRAIL receptor expression, we performed RNAi to knock down TRAIL receptor (DR4 and DR5) expression. The siRNAs were transfected into cells using Lipofectamine ${ }^{\mathrm{TM}}$ RNAiMAX Transfection Reagent (Invitrogen, Carlsbad, CA). The siRNAs used for gene knockdown were as follows: On-Target SMARTpool DR4 siRNA, On-Target SMARTpoo DR5 siRNA (Dharmacon, Inc., Lafayette, $C O$ ). siGENOME Non-Targeting siRNA \#1 (Dharmacon, Inc., Lafayette, $\mathrm{CO}$ ) was used as a negative control for siRNA-mediated knockdown. The knockdown of each gene was confirmed by real-time PCR and the extent of apoptosis was confirmed by Caspase-Glo 3/7 assay.

Statistics

Data analysis was carried out using paired $t$-test. Data were expressed as mean \pm SD of at least three sample replicates, unless stated otherwise.

\section{Results}

Inactivated HIV increases apoptosis in JFH1-infected Huh7.5.1 cells

To assess whether HCV and HIV induce apoptosis in hepatocytes, we performed a quantitative caspase activity assay and Western blot in Huh7.5.1 cells, JFH1-infected Huh7.5.1 cells, Huh7.5.1 cells grown in the presence of inactivated HIV (CCR5- and CXCR4-tropic strains),and JFH1-infected Huh7.5.1 cells grown in the presence of inactivated HIV (referred to as JFH1-infected/ inactivated-HIV treated Huh7.5.1 cells). Using the Caspase-Glo 3/7 assay, we examined the apoptotic effects of JFH1 HCV and heat-inactivated HIV on Huh7.5.1 cells.

Both JFH1 infection and inactivated HIV increased caspase 3/7 activity in Huh7.5.1 cells compared to uninfected controls (Fig. 2) ( $p<0.01$ for each). Furthermore, JFH1-infected/inactivated-HIV, both CCR5- and CXCR4-tropic strains, produced a greater than 2-fold increase of caspase 3/7 activity compared to either JFH1infected or inactivated-HIV Huh7.5.1 cells (Fig. 2) $(p<0.05$ for each). Western blot for cleaved PARP demonstrated that both JFH1-infected and inactivated-HIV Huh7.5.1 cells increased cleaved PARP expression compared to negative controls (Fig. 3) $(p<0.01$ for each); the expression of cleaved PARP was further increased in JFH1-infected/inactivated-HIV Huh7.5.1 cells $(p<0.05$ for each). From these experiments, we demonstrate that HIV increases HCV-induced hepatocyte apoptosis, and that HIV indirectly promotes hepatocyte apoptosis.

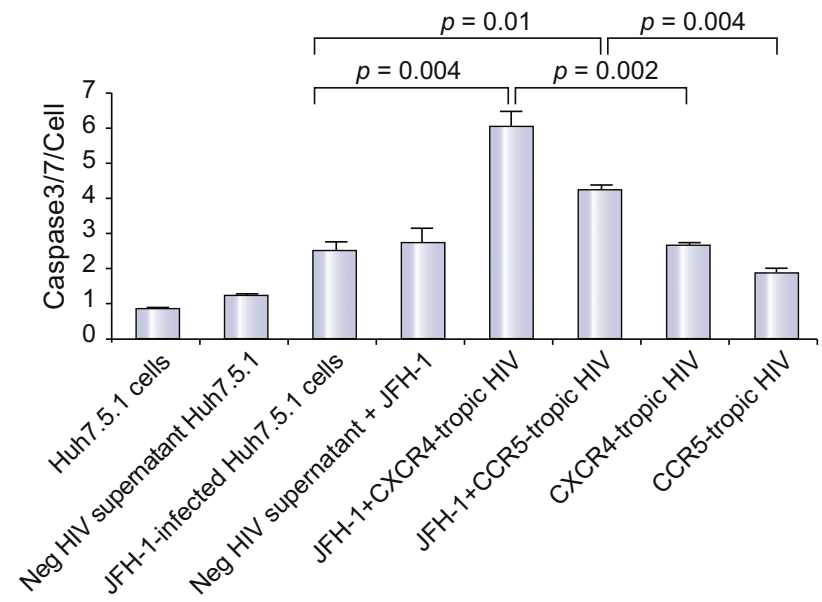

Fig. 2. Increased caspase 3/7 activity in JFH1-infected, heat-inactivated HIVtreated Huh7.5.1 cells compared to JFH1-infected or HIV-treated Huh7.5.1 cells alone. To assess the effects of HCV and HIV infection on apoptosis, 10,000 cells/well of Huh7.5.1 cells were added to 96 -well plates in $100 \mu \mathrm{l}$ of $10 \%$ FBS DMEM, $24 \mathrm{~h}$ before transfection. Transfection of JFH1 and incubation with heatinactivated HIV were performed simultaneously. Caspase-Glo 3/7 reagent was added to each well $72 \mathrm{~h}$ later. Each treatment was performed in triplicate. Caspase 3/7 activity was increased by over 2-fold in JFH1-infected, heatinactivated HIV-treated Huh7.5.1 cells compared to JFH1-infected or HIV-treated Huh7.5.1 cells ( $p<0.05$ for each). $Y$ axis refers to caspase $3 / 7$ activity per cell. Lane \#1 Huh7.5.1, \#2 Huh7.5.1 + negative supernatant HIV, \#3 JFH1, \#4 JFH1 + negative supernatant HIV, \#5 JFH1 + CXCR4 tropic HIV, \#6 JFH1 + CCR5 tropic HIV, \#7 CXCR4 tropic HIV, \#8 CCR5 tropic HIV. 


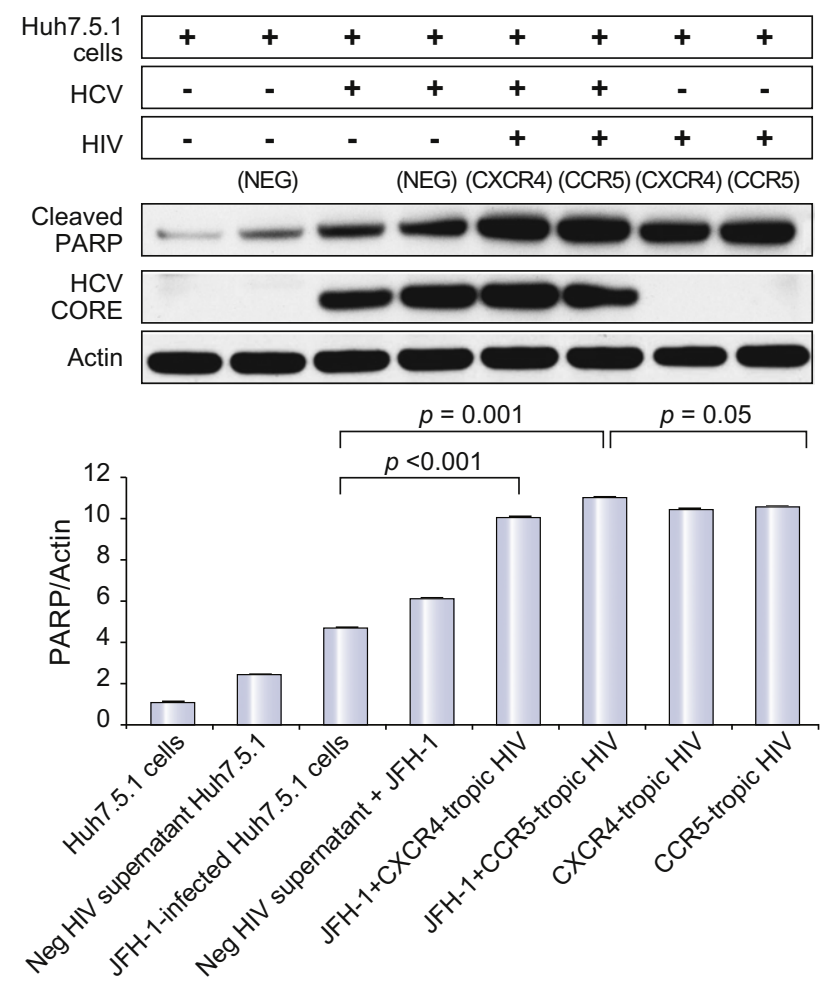

Fig. 3. Expression of cleaved PARP was increased in JFH1-infected, heatinactivated HIV-treated Huh7.5.1 cells compared to JFH1-infected or HIVtreated Huh7.5.1 cells alone. To assess apoptosis in HCV and HIV co-infected Huh7.5.1 cells, we monitored cleaved PARP, HCV core, and $\beta$-actin levels by Western blot and corresponding densitometry. We confirmed that expression of cleaved PARP was increased in JFH1-infected, heat-inactivated HIV-treated Huh7.5.1 cells compared to JFH1-infected or HIV-treated Huh7.5.1 cells alone ( $p<0.05$ for each). Lane \#1 Huh7.5.1, \#2 Huh7.5.1 + negative supernatant HIV, \#3 JFH1, \#4 JFH1 + negative supernatant HIV, \#5 JFH1 + CXCR4-tropic HIV, \#6 JFH1 + CCR5-tropic HIV, \#7 CXCR4-tropic HIV, \#8 CCR5-tropic HIV.

Increased expression of TRAIL receptor 1 and 2 is observed in HCVinfected Huh7.5.1 cells in the presence of HIV compared to HCV-

infected or HIV-treated Huh7.5.1 cells

To further assess the molecular mechanisms of apoptosis induced by these viruses, we examined known mediators of apoptotic signaling, specifically TRAIL and TRAIL receptor 1, 2 (DR4, DR5). We first measured levels of TRAIL receptor 1 (DR4), 2 (DR5), and TRAIL using real-time PCR. We found that DR4 and DR5 mRNA levels were increased in HCV-infected Huh7.5.1 cells in the presence of heat-inactivated HIV compared to Huh7.5.1 cells infected with JFH1 or exposed to heat-inactivated HIV alone (Fig. 4A and B). DR5 was significantly increased in JFH1-infected, heat-inactivated HIV-treated Huh7.5.1 cells compared to JFH1 or heat-inactivated HIV-treated Huh7.5.1 cells alone $(p<0.05)$ [1.23-fold (vs HCV), 2.41-fold ( $v s$ HIV)] and DR4 was moderately increased $(p=0.05)$ (1.37-fold ( $v s \mathrm{HCV}), 2.48$-fold ( $v s$ HIV). In the case of TRAIL, mRNA levels were decreased in the presence of HCV compared to Huh7.5.1 cells and HIV-incubated Huh7.5.1 cells (Fig. 4C). For further evaluation of TRAIL signaling, we performed Western blot for DR4, DR5, and TRAIL. As shown in Fig. 5, DR4 and DR5 induction was observed in HCV-infected Huh7.5.1 cells in the presence of heat-inactivated HIV compared to either JFH1-infected or heat-inactivated HIV-treated Huh7.5.1 cells

\section{JOURNAL OF HEPATOLOGY}

[DR4 $(p<0.01)(2.02$-fold ( $v s$ HCV), 1.80-fold ( $v s$ HIV)] [DR5 $(p<0.01)$ (1.55-fold ( $v s$ HCV), 1.50-fold ( $v s$ HIV)]. Protein expression of TRAIL was decreased in the presence of HCV. These results suggest that HIV increases HCV-induced hepatocyte apoptosis, and that this increase in apoptosis is induced by up-regulation of the TRAIL receptors DR4 and DR5.

HCV-HIV induced apoptosis is dependent on caspases within the mitochondrial pathway

To further delineate the intracellular apoptotic pathway modulated by HCV and HIV, we investigated the activation of caspase pathways during apoptosis using several caspase inhibitors. We performed Caspase-Glo 3/7 assay after pre-incubation with several caspase inhibitors. The pan-caspase inhibitor zVAD-fmk decreased HCV, HIV, or HCV-HIV-induced caspase 3/7 activity by over 4 -fold $(p<0.01)$. A caspase- 8 inhibitor blocked HCV, HIV, or HCV-HIV-induced apoptosis to the same degree as zVAD-fmk. In addition, a caspase- 9 inhibitor blocked apoptosis induced by HCV, HIV, or HCV-HIV comparably to pancaspase or caspase-8 inhibition (Fig. 6). Taken together, these data indicate that HCV, HIV, or HCV-HIV-induced apoptosis are dependent on both extrinsic caspases and caspases related to the mitochondrial pathway.

\section{Analysis of Bid cleavage and cytochrome C levels}

To confirm the activation of the mitochondrial pathway upon HCV-HIV infection of Huh7.5.1 cells, we performed Western blot of the mitochondrial apoptosis activating protein Bid and cytochrome $C$. While HCV alone induced the activation of Bid cleavage and cytochrome $C$ release, addition of HIV substantially augmented this induction (3.5-fold for BID, 1.6-fold for cytochrome C, Fig. 7). These data confirm that HCV-HIV-related apoptosis utilizes the mitochondrial pathway.

\section{HCV-HIV induced apoptosis is decreased by DR4 and DR5 siRNA}

To demonstrate that HCV and HIV-induced increases in apoptosis are mediated by DR4 and DR5, we performed siRNA-mediated knockdown of DR4 and DR5 (Fig. 8). We found that HCV-HIVinduced apoptosis was blocked by DR4- and DR5-specific siRNAs, but not by negative control siRNAs (Fig. 8A). These data confirm that HCV-HIV-induced apoptosis is dependent on DR4 and DR5 up-regulation. The presence of residual apoptosis that is not eliminated by DR4 or DR5 siRNAs in the presence of HIV or HIV-HCV infected cells, raises the possibility that additional non-DR4, -DR5 pathways contribute to apoptosis.

\section{Discussion}

Fibrogenesis is a relatively late event in chronic liver injury, and occurs as a consequence of activation of hepatic stellate cells (HSC) and excessive deposition of extracellular matrix, under conditions of persistent inflammation.

The first phase of liver injury, however, independent of the etiology, is almost always characterized by increased hepatocyte apoptosis. Since for many years apoptosis has been considered a mechanism of cell death not associated with an inflammatory 


\section{Research Article}
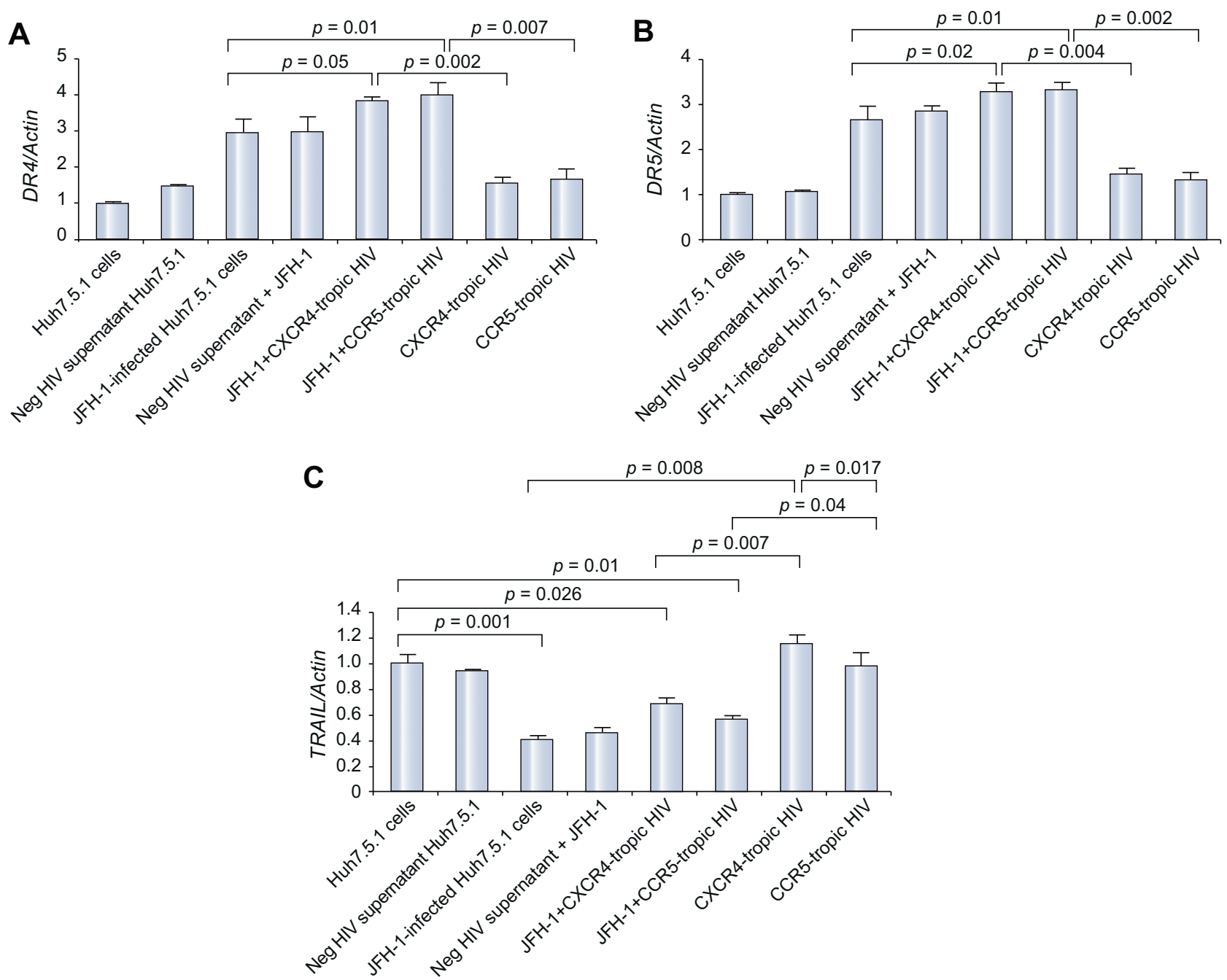

Fig. 4. Increased DR4 and DR5 mRNA levels in HCV-infected Huh7.5.1 cells in the presence of heat-inactivated HIV compared to JFH1-infected or HIV-treated Huh7.5.1 cells. Total cellular RNA was harvested, and DR4 (A), DR5 (B), TRAIL (C) and $\beta$-actin mRNA levels were quantified by real-time PCR. Each treatment was performed in triplicate. DR4, DR5, and TRAIL levels were normalized to $\beta$-actin levels to calculate DR4, DR5, and TRAIL/actin arbitrary units. Real-time PCR demonstrates increased relative DR4 and DR5 mRNA levels in HCV-infected, HIV-treated Huh7.5.1 cell compared to JFH1-infected or HIV treated Huh7.5.1 cells. DR5 mRNA levels were significantly increased in HCV-infected Huh7.5.1 cells in the presence of heat-inactivated HIV compared to JFH1-infected or HIV-treated Huh7.5.1 cells ( $p<0.05$ ) and DR4 mRNA was moderately increased $(p=0.05)$. In the case of TRAIL, mRNA levels were decreased in the presence of HCV. Lane \#1 Huh7.5.1, \#2 Huh7.5.1 + negative supernatant HIV, \#3 JFH1, \#4 JFH1 + negative supernatant HIV, \#5 JFH1 + CXCR4-tropic HIV, \#6 JFH1 + CCR5 tropic HIV, \#7 CXCR4-tropic HIV, \#8 CCR5-tropic HIV.

response, these two phases have not been linked until recently [14].

Because of their shared routes of transmission, co-infection with HCV is increasingly a major cause of morbidity and mortality among $\mathrm{HIV}^{+}$persons [4]. For instance, it is well established that $\mathrm{HIV}^{+} / \mathrm{HCV}^{+}$persons experience more progressive liver fibrosis and higher rates of cirrhosis, liver failure, and death than $\mathrm{HIV}^{-} / \mathrm{HCV}^{+}$persons [33-38] despite successful control of HIV with antiretroviral therapy.

The mechanisms underlying the accelerated evolution of $\mathrm{CHC}$ in $\mathrm{HCV} / \mathrm{HIV}$ coinfection are not known. We have previously shown [30] that the addition of heat-inactivated HIV indirectly regulates $\mathrm{HCV}$ replication and, ultimately, the fibrogenic actions of HCV, by augmenting TGF- $\beta 1$ expression in HCV-infected hepatocytes. Furthermore, this increase in HCV replication was abrogated by pre-incubation with neutralizing antibody to CXCR4 and CCR5. These data, thereby, provided evidence for an indirect effect of HIV virus signaling through engagement of its co-receptor on the surface of Huh7.5.1 cells rather than through the infection itself. Another possible explanation for accelerated fibrogenesis is enhanced hepatocyte apoptosis. In the current study, we now extend our previous findings to demonstrate that heat-inactivated HIV can also augment HCV-mediated apoptosis. The observed increased apoptosis in JFH1-infected, heat-inactivated HIV-treated Huh7.5.1 cells, compared to JFH1 or heat-inactivated HIV-treated Huh7.5.1 cells alone, may contribute to more progressive liver fibrosis in HCV-HIV coinfected patients. Because we also found that HIV alone can moderately induce hepatocyte apoptosis, these data suggest that HIV predisposes the liver to a multitude of second hits, including HCV and HBV co-infection, both of whose natural history are accelerated in HIV-infected persons. 


\section{JOURNAL OF HEPATOLOGY}
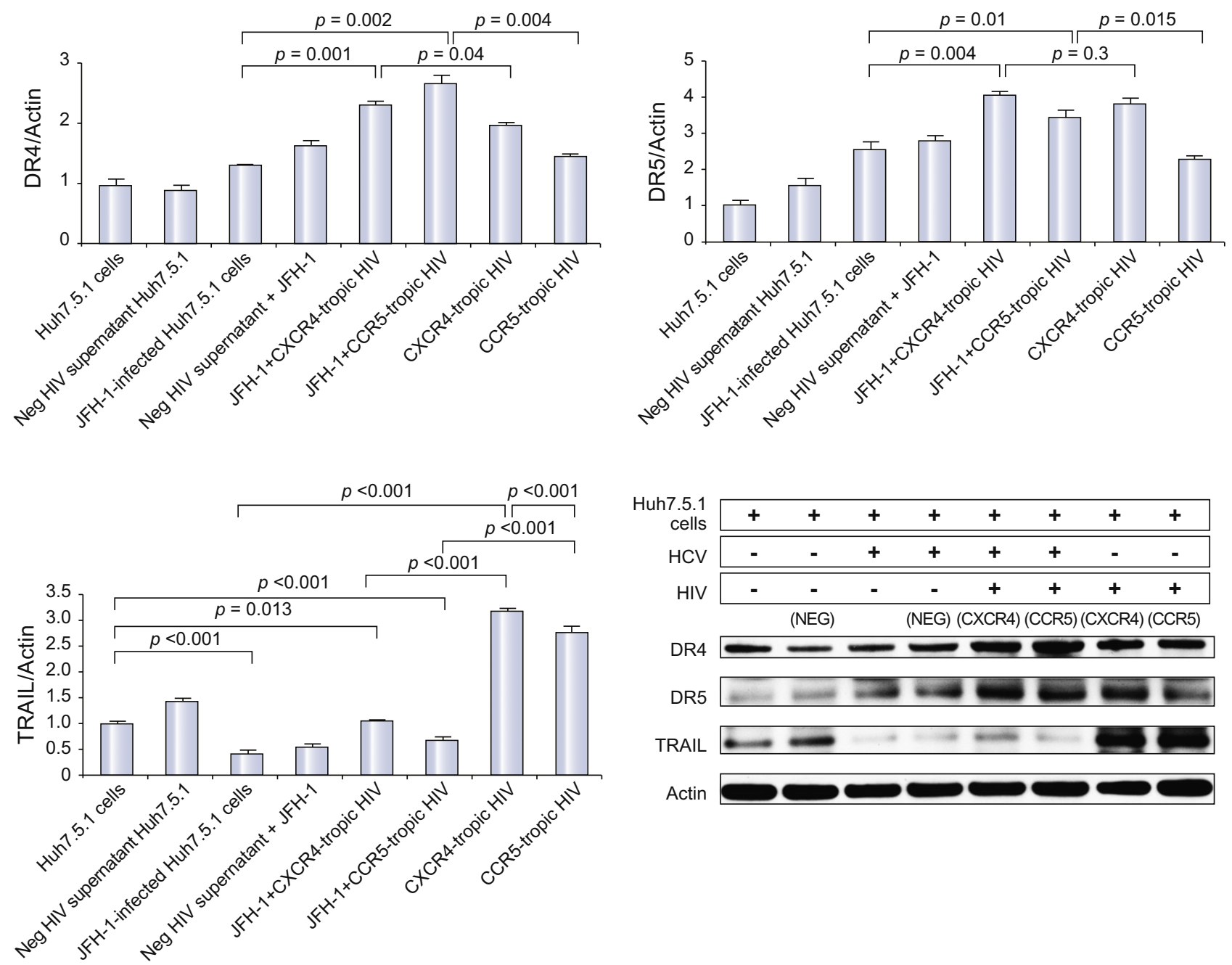

Fig. 5. Increased DR4 and DR5 protein expression in HCV-infected Huh7.5.1 cells in the presence of heat-inactivated HIV compared to JFH1-infected or HIV-treated Huh7.5.1 cells. Protein lysates were harvested for Western blot for DR4, DR5 and TRAIL. Corresponding densitometry was performed. We found that protein expression of DR4 and DR5 was increased in JFH1-infected, heat-inactivated HIV-treated Huh7.5.1 cells compared to JFH1 or HIV-treated Huh7.5.1 cells alone ( $p<0.01$ for each). Protein expression of TRAIL was decreased in the presence of HCV. Lane\#1 Huh7.5.1, \#2 Huh7.5.1 + negative supernatant HIV, \#3 JFH1, \#4 JFH1 + negative supernatant HIV, \#5 JFH1 + CXCR4-tropic HIV, \#6 JFH1 + CCR5-tropic HIV, \#7 CXCR4-tropic HIV, \#8 CCR5-tropic HIV.

Several studies have postulated an autocrine TRAIL-dependent apoptosis of hepatocytes [39,40]. Other studies suggest that a TRAIL autocrine loop does not play a major role in TRAILinduced sensitization [41]. HCV-dependent up-regulation of TRAIL and apoptosis induction in a novel hepatoma cell line have been described [42]. Others [43] have described that HIV selectively up-regulates TRAIL-R2 expression in hepatocytes and confers an acquired sensitivity to TRAIL-mediated apoptosis.

In the present study, we report that HCV-infected, HIV-treated Huh7.5.1 cells express higher levels of TRAIL receptor (DR4 and DR5), and apoptosis than do HCV or HIV-treated Huh7.5.1 cells alone. However, TRAIL was decreased in the presence of HCV, suggesting that an autocrine loop is not operative. Rather, our data suggest either that increased DR4 or DR5 expression or sensitivity may compensate for decreases in TRAIL release or that additional death receptor pathways contribute to this decreased release. The finding that circulating TRAIL levels are increased in HIV monoinfection [44] compared to no infection or HCV monoinfection suggests another basis for the predisposition of the HIV-infected liver to enhanced injury from other sources.

We investigated the involvement of caspase pathways using several caspase inhibitors. Lan et al. [41] previously demonstrated that the mitochondrially-activated caspase pathway is triggered in HCV infection by the use of selective caspase inhibitors. We also sought to assess the operative pathways in HCVHIV co-infection using these inhibitors. Our results indicate that the HCV-HIV mediated apoptosis signal is transduced from DR4 and DR5 through activation of caspase 8 , which in turn activates the mitochondrial signaling pathway, whose involvement was confirmed by our demonstration of Bid cleavage and cytochrome $C$ activation after HCV and HIV coinfection. Furthermore, because a caspase- 8 inhibitor was equally as effective as pancaspase and caspase- 9 inhibitors, our data imply that TRAIL-mediated apoptosis engages both the caspase- 8 and the mitochondrial pathways.

Using an infectious tissue culture model, we provide evidence that HCV and HIV infection increases hepatocyte apoptosis 
Research Article
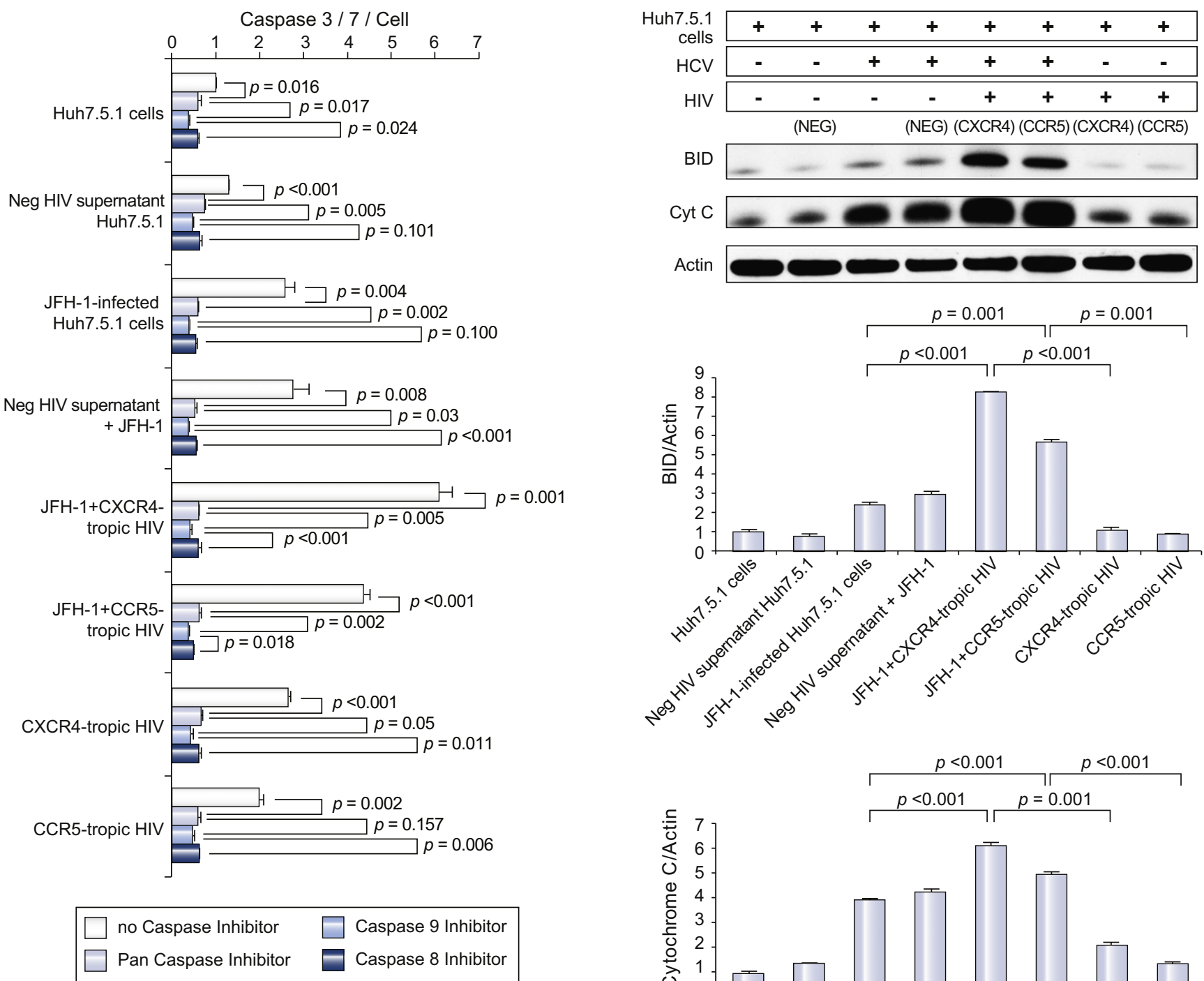

Fig. 6. HCV-HIV induced apoptosis is dependent on caspases related to the mitochondrial pathway. Naïve Huh7.5.1 cell and HCV and/or HIV treated Huh7.5.1 cells were incubated with $20 \mu \mathrm{M}$ of a variety of caspase inhibitors in 96well plates $(10,000$ cell/well) for $6 \mathrm{~h}$ prior to Caspase-Glo 3/7 assay. Inhibitors used included the pan-caspase inhibitor Z-VAD-FMK, the caspase 8 inhibitor ZIETD-FMK, and the caspase 9 inhibitor Z-LEHDFMK. The pan-caspase inhibitor zVAD-fmk decreased HCV, HIV, or HCV-HIV induced caspase $3 / 7$ activity by over 4 -fold $(p<0.01)$. The caspase 8 inhibitor blocked HCV, HIV, or HCV-HIV induced apoptosis to the same degree as that inhibited by zVAD-fmk. In addition, a caspase 9 inhibitor significantly blocked apoptosis induced by HCV, HIV, or HCVHIV comparably to the pancaspase and caspase 8 inhibitors. The $y$ axis refers to caspase 3/7 activity per cell. Lane \#1 Huh7.5.1, \#2 Huh7.5.1 + negative supernatant HIV, \#3 JFH1, \#4 JFH1 + negative supernatant HIV, \#5 JFH1 + CXCR4 tropic HIV, \#6 JFH1 + CCR5 tropic HIV, \#7 CXCR4-tropic HIV, \#8 CCR5-tropic HIV.

compared to HCV or HIV alone. We propose a model in which HCV and HIV each induces TRAIL receptors (DR4 and DR5), which in turn activate caspase 8 and the mitochondrial pathway. Our data further provide an additional plausible mechanism for the accelerated disease progression observed in HCV-HIV co-infection. Efforts to specifically inhibit this apoptotic program may be warranted to retard liver disease progression in co-infected persons.

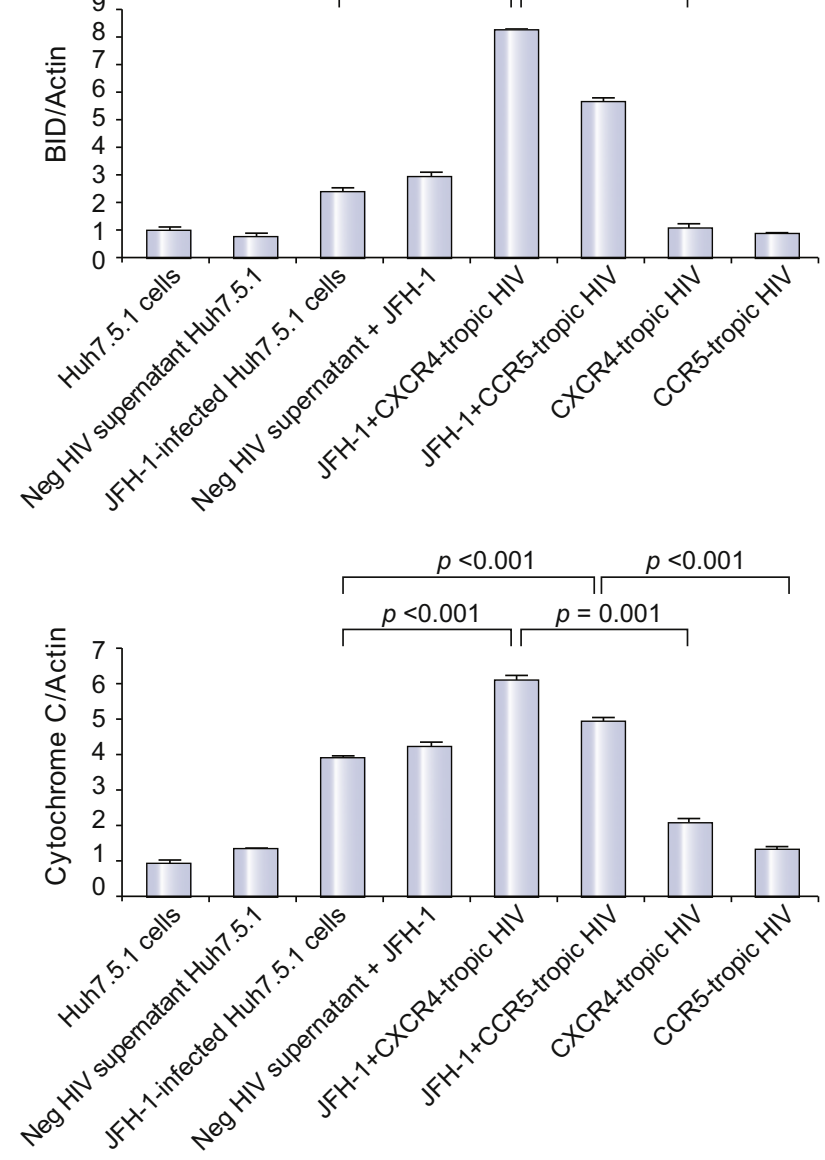

Fig. 7. Analysis of Bid cleavage and Cytochrome $\boldsymbol{C}$ levels. Protein lysates were harvested for Western blot analysis of the mitochondrial apoptosis activating protein Bid and cytochrome $C$. Corresponding densitometry was performed. While HCV alone induced the activation of Bid cleavage and cytochrome $C$ release, addition of heat-inactivated HIV substantially augmented this induction (3.47fold for BID, 1.60-fold for cytochrome $C$ by densitometry). Lane \#1 Huh7.5.1, \#2 Huh7.5.1 + negative supernatant HIV, \#3 JFH1, \#4 JFH1 + negative supernatant HIV, \#5 JFH1 + CXCR4-tropic HIV, \#6 JFH1 + CCR5-tropic HIV, \#7 CXCR4-tropic HIV, \#8 CCR5-tropic HIV.

\section{Conflict of interest}

The authors who have taken part in this study declared that they do not have anything to disclose regarding funding or conflict of interest with respect to this manuscript. 


\section{JOURNAL OF HEPATOLOGY}
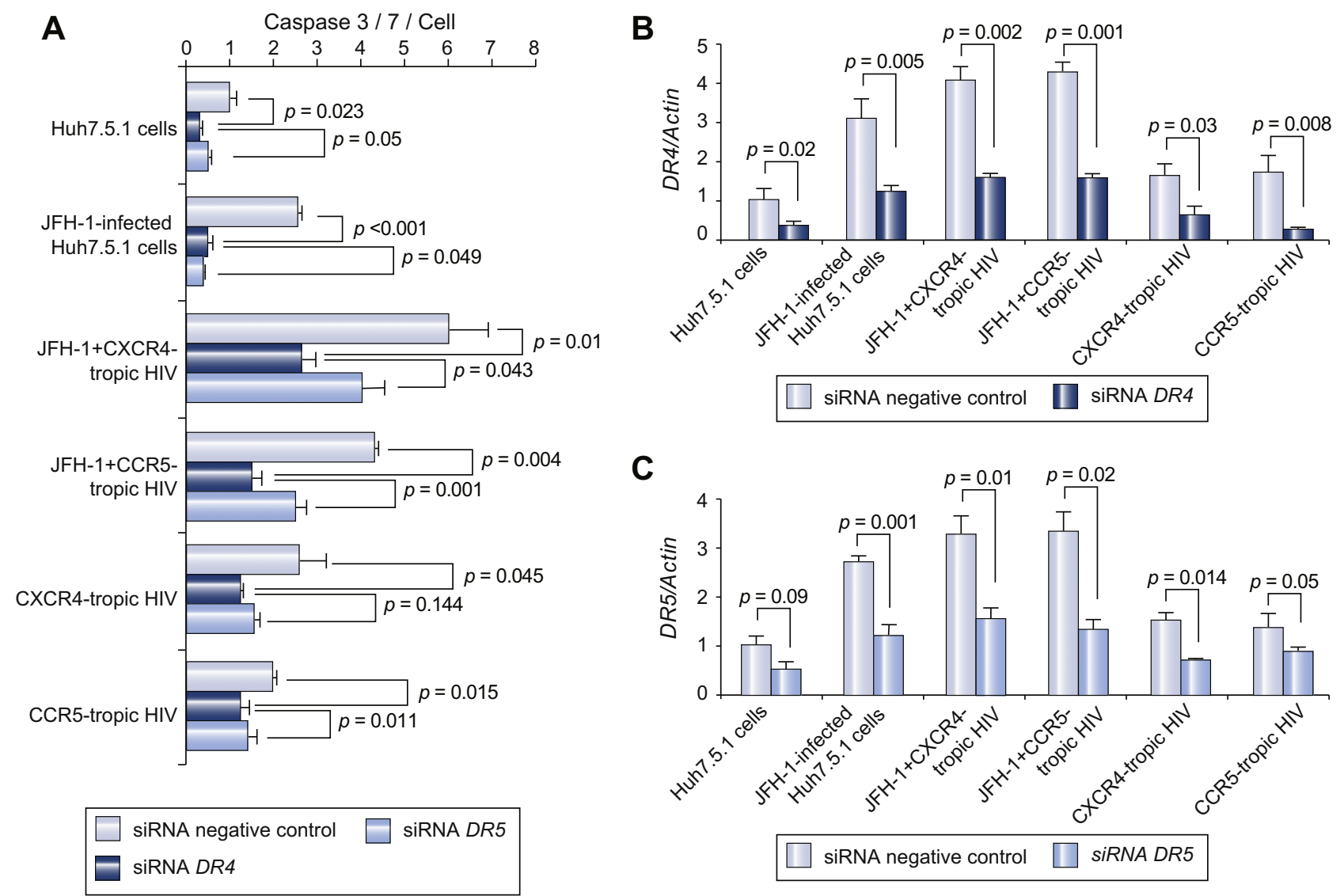

Fig. 8. Effects of DR4 and DR5 knockdown on HCV-HIV induced apoptosis. The indicated siRNAs were transfected into HCV-HIV infected Huh7.5.1 cells in 96-well plate. The siRNAs used for gene knockdown included DR4-, DR5-targeted, or negative control siRNAs. Real-time PCR assay was performed to measure DR4 and DR5 expression levels in these siRNA-transfected cells. (B and C) DR4 and DR5 mRNA levels were decreased by over 2-fold in DR4- and DR5-targeted siRNA-transfected cells compared to negative siRNA-transfected cells. (A) Caspase-Glo 3/7 staining revealed that HCV-HIV induced apoptosis was suppressed by nearly 2-fold in DR4- and DR5-targeted siRNAtransfected cells compared to negative control siRNA-transfected cells. Lane \#1 Huh7.5.1, \#2 JFH1, \#3 JFH1 + CXCR4-tropic HIV, \#4 JFH1 + CCR5-tropic HIV, \#5 CXCR4tropic HIV, \#6 CCR5-tropic HIV.

\section{Financial support}

This work was supported in part by NIH grants AI069939 and AI082630 (RTC).

\section{Acknowledgments}

We gratefully acknowledge: Dr. Ralf Bartenschlager, University, Heidelberg, Germany; and Dr. Takaji Wakita, Second Department of Virology, National Institute of Infectious Diseases, Tokyo, Japan (infectious HCV virus JFH1 DNA construct); and Dr. Francis Chisari, Scripps Institute, CA (Huh7.5.1 cells). This work was support by NIH Grants R01 AI069939 and U19 AI082630 (to R.T.C.).

\section{References}

[1] Alter MJ. Epidemiology of viral hepatitis and HIV co-infection. J Hepatol 2006;44:S6-9.

[2] Lauer GM, Walker BD. Hepatitis $\mathrm{C}$ virus infection. $\mathrm{N}$ Engl J Med 2001;345:41-52.

[3] Munshi N, Balasubramanian A, Koziel M, Ganju RK, Groopman JE. Hepatitis C and human immunodeficiency virus envelope proteins cooperatively induce hepatocytic apoptosis via an innocent bystander mechanism. J Infect dis 2003;188:1192-1204.
[4] Bica I, McGovern B, Dhar R, Stone D, McGowan K, Scheib R, et al. Increasing mortality due to endstage liver disease in patients with human immunodeficiency virus infection. Clin Infect Dis 2001;32:492-497.

[5] Cao YZ, Dieterich D, Thomas PA, Huang YX, Mirabile M, Ho DD. Identification and quantitation of HIV-1 in the liver of patients with AIDS. AIDS 1992;6:65-70.

[6] Lefkowitch JH. Pathology of AIDS-related liver disease. Dig Dis 1994; 12:321-330.

[7] Housset C, Boucher O, Girard PM, Leibowitch J, Saimot AG, Bréchot C, et al. Immunohistochemical evidence for human immunodeficiency virus-1 infection of liver Kupffer cells. Hum Pathol 1990;21:404-408.

[8] Rust C, Gores GJ. Apoptosis and liver disease. Am J Med 2000;108:567-574.

[9] Jaeschke H, Gores GJ, Cederbaum AI, Hinson JA, Pessayre D, Lemasters JJ. Mechanisms of hepatotoxicity. Toxicol Sci 2002;65:166-176.

[10] Lawson JA, Fisher MA, Simmons CA, Farhood A, Jaeschke H. Parenchymalcell apoptosis as a signal for sinusoidal sequestration and transendothelial migration of neutrophils in murine models of endotoxin and Fas-antibodyinduced liver injury. Hepatology 1998;28:761-767.

[11] Faouzi S, Burckhardt BE, Hanson JC, Campe CB, Schrum LW, Rippe RA. AntiFas induces hepatic chemokines and promotes inflammation by an NFkappa B-independent, caspase-3-dependent pathway. J Biol Chem 2001;276:49077-49082.

[12] Canbay A, Friedman S, Gores GJ. Apoptosis: the nexus of liver injury and fibrosis. Hepatology 2004;39:273-278.

[13] Bantel H, Lugering A, Poremba C, Lugering N, Held J, Domschke W, et al. Caspase activation correlates with the degree of inflammatory liver injury in chronic hepatitis C virus infection. Hepatology 2001;34:758-767. 


\section{Research Article}

[14] Walsh MJ, Vanags DM, Clouston AD, Richardson MM, Purdie DM, Jonsson JR, et al. Steatosis and liver cell apoptosis in chronic hepatitis C: a mechanism for increased liver injury. Hepatology 2004;39:1230-1238.

[15] Feldstein AE, Canbay A, Angulo P, Taniai M, Burgart LJ, Lindor KD, et al Hepatocyte apoptosis and fas expression are prominent features of human nonalcoholic steatohepatitis. Gastroenterology 2003;125:437-443.

[16] MacFarlane M, Ahmad M, Srinivasula SM, Fernandes-Alnemri T, Cohen GM, Alnemri ES. Identification and molecular cloning of two novel receptors for the cytotoxic ligand TRAIL. J Biol Chem 1997;272:25417-25420.

[17] Walczak H, Degli-Esposti MA, Johnson RS, Smolak PJ, Waugh JY, Boiani N, et al. TRAIL-R2: a novel apoptosis-mediating receptor for TRAIL. EMBO J. 1997; 16:5386-5397.

[18] Wiley SR, Schooley K, Smolak PJ, Din WS, Huang CP, Nicholl JK, et al Identification and characterization of a new member of the TNF family that induces apoptosis. Immunity 1995;3:673-682.

[19] Pitti RM, Marsters SA, Ruppert S, Donahue CJ, Moore A, Ashkenazi A. Induction of apoptosis by Apo-2 ligand, a new member of the tumor necrosis factor cytokine family. J Biol Chem 1996;271:12687-12690.

[20] Ashkenazi A, Pai RC, Fong S, Leung S, Lawrence DA, Marsters SA, et al. Safety and antitumor activity of recombinant soluble Apo2 ligand. J Clin Invest 1999;104:155-162.

[21] Walczak H, Miller RE, Ariail K, Gliniak B, Griffith TS, Kubin M, et al Tumoricidal activity of tumor necrosis factor-related apoptosis-inducing ligand in vivo. Nat Med 1999;5:157-163.

[22] Lawrence D, Shahrokh Z, Marsters S, Achilles K, Shih D, Mounho B, et al Differential hepatocyte toxicity of recombinant Apo2L/TRAIL versions. Nat Med 2001;7:383-385

[23] Hersey P, Zhang XD. How melanoma cells evade trail-induced apoptosis. Nat Rev Cancer 2001;1:142-150.

[24] Kischkel FC, Lawrence DA, Tinel A, LeBlanc H, Virmani A, Schow P, et al Death receptor recruitment of endogenous caspase-10 and apoptosis initiation in the absence of caspase-8. J Biol Chem 2001;276:46639-46646.

[25] Wang J, Chun HJ, Wong W, Spencer DM, Lenardo MJ. Caspase-10 is an initiator caspase in death receptor signaling. Proc Natl Acad Sci USA 2001;98:13884-13888.

[26] Yamada H, Tada-Oikawa S, Uchida A, Kawanishi S. TRAIL causes cleavage of bid by caspase- 8 and loss of mitochondrial membrane potential resulting in apoptosis in BJAB cells. Biochem Biophys Res Commun 1999;265:130-133.

[27] Wakita T, Pietschmann T, Kato T, Date T, Miyamoto M, Zhao Z, et al. Production of infectious hepatitis $C$ virus in tissue culture from a cloned viral genome. Nat Med 2005;11:791-796.

[28] Brockman MA, Tanzi GO, Walker BD, Allen TM. Use of a novel GFP reporter cell line to examine replication capacity of CXCR4- and CCR5-tropic HIV-1 by flow cytometry. J Virol Methods 2006;131:134-142.

[29] Gartner S, Markovits P, Markovitz DM, Kaplan MH, Gallo RC, Popovic M. The role of mononuclear phagocytes in HTLV-III/LAV infection. Science 1986;233:215-219.

[30] Lin W, Weinberg EM, Tai AW, Peng LF, Brockman MA, Kim KA, et al. HIV increases HCV replication in a TGF- $\beta 1$-dependent manner. Gastroenterology 2008; 134:803-811.
[31] Lin W, Choe WH, Hiasa Y, Kamegaya Y, Blackard JT, Schmidt EV, et al. Hepatitis $C$ virus expression suppresses interferon signaling by degrading STAT1. Gastroenterology 2005;128:1034-1041.

[32] Oliver FJ, de la Rubia G, Rolli V, Ruiz-Ruiz MC, de Murcia G, Murcia JM. Importance of poly(ADP-ribose) polymerase and its cleavage in apoptosis. Lesson from an uncleavable mutant. J Biol Chem 1998;273:33533-33539.

[33] Martinez-Sierra C, Arizcorreta A, Díaz F, Roldán R, Martín-Herrera L, PérezGuzmán E, et al. Progression of chronic hepatitis $C$ to liver fibrosis and cirrhosis in patients coinfected with hepatitis $\mathrm{C}$ virus and human immunodeficiency virus. Clin Infect Dis 2003;36:491-498.

[34] Lesens O, Deschênes M, Steben M, Bélanger G, Tsoukas CM. Hepatitis C virus is related to progressive liver disease in human immunodeficiency viruspositive hemophiliacs and should be treated as an opportunistic infection. J Infect Dis 1999;179:1254-1258.

[35] Ragni MV, Belle SH. Impact of human immunodeficiency virus infection on progression to end-stage liver disease in individuals with hemophilia and hepatitis C virus. J Infect Dis 2001;183:1112-1115.

[36] Tedaldi EM, Baker RK, Moorman AC, Alzola CF, Furhrer J, McCabe RE, et al. HIV Outpatient Study (HOPS) Investigators. Influence of coinfection with hepatitis $\mathrm{C}$ virus on morbidity and mortality due to human immunodeficiency virus infection in the era of highly active antiretroviral therapy. Clin Infect Dis 2003;36:363-367.

[37] Puoti M, Bonacini M, Spinetti A, Putzolu V, Govindarajan S, Zaltron S, et al. HIV-HCV coinfection study group. Liver fibrosis progression is related to CD4 cell depletion in patients coinfected with hepatitis $C$ virus and human immunodeficiency virus. J Infect Dis 2001:183:134-137.

[38] Benhamou Y, Bochet M, Di Martino V, Charlotte F, Azria F, Coutellier A, et al. Liver fibrosis progression in human immunodeficiency virus and hepatitis $C$ virus coinfected patients. The Multivirc Group. Hepatology 1999;30:1054-1058.

[39] Mundt B, Kuhnel F, Zender L, Paul Y, Tillmann H, Trautwein C, et al. Involvement of TRAIL and its receptors in viral hepatitis. FASEB J 2003;17:94-96.

[40] Volkmann X, Fischer U, Bahr MJ, Ott M, Lehner F, Macfarlane M, et al. Increased hepatotoxicity of tumor necrosis factor-related apoptosis-inducing ligand in diseased human liver. Hepatology 2007;46:1498-1508.

41] Lan L, Gorke S, Rau SJ, Zeisel MB, Hildt E, Himmelsbach K, et al. Hepatitis C virus infection sensitizes human hepatocytes to TRAIL-induced apoptosis in a caspase 9-dependent manner. J Immunol 2008;181:4926-4935.

[42] Zhu H, Dong H, Eksioglu E, Hemming A, Cao M, Crawford JM, et al. Hepatitis $C$ virus triggers apoptosis of a newly developed hepatoma cell line through antiviral defense system. Gastroenterology 2007;133:1649-1659.

[43] Babu CK, Suwansrinon K, Bren GD, Badley AD, Rizza SA. HIV induces TRAIL sensitivity in hepatocytes. PLoS ONE 2009;4:e4623.

[44] Herbeuval JP, Boasso A, Grivel JC, Hardy AW, Anderson SA, Dolan MJ, et al. TNF-related apoptosis-inducing ligand (TRAIL) in HIV-1-infected patients and its in vitro production by antigen-presenting cells. Blood 2005;105:2458-2464. 